\title{
On the Convergence of Solutions of Globally Modified Navier-Stokes Equations with Delays to Solutions of Navier-Stokes Equations with Delays
}

\author{
Pedro Marín-Rubio, José Real* \\ Departamento de Ecuaciones Diferenciales y Análisis Numérico \\ Universidad de Sevilla, Apdo. de Correos 1160, 41080-Sevilla, Spain \\ e-mail:pmr@us.es, jreal@us.es \\ Antonio M. Márquez-Durán \\ Departamento de Economía, Métodos Cuantitativos e Historia Económica \\ Universidad Pablo de Olavide, Ctra. de Utrera, Km. 1, 41013-Sevilla, Spain \\ e-mail:ammardur@upo.es \\ Received 5 December 2010 \\ Communicated by Peter E. Kloeden
}

\begin{abstract}
We prove that under suitable assumptions, from a sequence of solutions of Globally Modified Navier-Stokes equations with delays we can extract a subsequence which converges in an adequate sense to a weak solution of a three-dimensional Navier-Stokes equation with delays. An additional case with a family of different delays involved in the approximating problems is also discussed.
\end{abstract}

2010 Mathematics Subject Classification. 35Q30, 35K55, 35R10.

Key words. Globally Modified Navier-Stokes equations; three-dimensional Navier-Stokes equations; Navier-Stokes equations with delays.

${ }^{*}$ This work was partially supported by the Spanish Ministerio de Ciencia e Innovación, Project MTM2008-00088, and the Consejería de Innovación, Ciencia y Empresa (Junta de Andalucía), Proyecto de Excelencia P07-FQM-02468. 


\section{Introduction}

Let $\Omega \subset \mathbb{R}^{3}$ be an open bounded set with regular boundary $\Gamma$. For each $N \in(0,+\infty)$ let us define $F_{N}:[0,+\infty) \rightarrow(0,1]$ by

$$
F_{N}(r):=\min \left\{1, \frac{N}{r}\right\}, \quad r \in[0,+\infty)
$$

and consider the following system of globally modified Navier-Stokes equations in $\Omega$, with delays and homogeneous Dirichlet boundary condition:

$$
\left\{\begin{array}{l}
\frac{\partial u}{\partial t}-v \Delta u+F_{N}(\|u\|)[(u \cdot \nabla) u]+\nabla p=G(t, u(t-\rho(t))) \text { in }(0, T) \times \Omega, \\
\nabla \cdot u=0 \text { in }(0, T) \times \Omega, \\
u=0 \text { on }(0, T) \times \Gamma \\
u(0, x)=u_{0}(x), \quad x \in \Omega, \\
u(t, x)=\phi(t, x), \text { in }(-h, 0) \times \Omega
\end{array}\right.
$$

where $u$ is the unknown velocity field of the fluid, $p$ is the unknown pressure, and we have given $v>0$, the kinematic viscosity, $T>0$ a final time, the external force term $G(t, u(t-\rho(t)))$, which depends on the value $u(t-\rho(t))$, where $0 \leq \rho(t) \leq h$, with $h>0$, is a delay function, $u_{0}$ an initial velocity field, and $\phi$ a velocity field defined in $(-h, 0)$.

The globally modified Navier-Stokes equations, in the case without delays, were introduced and studied in [1] (see also [2, 3, 10, 11, 12, 21] and the review paper [9]). However, there are situations in which the model is better described if some terms containing delays appear in the equations.

The system (1.1) has been studied in [4], where existence, uniqueness and asymptotic behaviour of a solution $u^{(N)}=u^{(N)}\left(\cdot ; u_{0}, \phi\right)$ were analyzed (see also $[16,17,18]$ for the case of infinite delays with and without modification).

This system is a modification of the the following system of Navier-Stokes equations, with delays and homogeneous Dirichlet boundary condition:

$$
\left\{\begin{array}{l}
\frac{\partial u}{\partial t}-v \Delta u+(u \cdot \nabla) u+\nabla p=G(t, u(t-\rho(t))) \text { in }(0, T) \times \Omega \\
\nabla \cdot u=0 \text { in }(0, T) \times \Omega \\
u=0 \text { on }(0, T) \times \Gamma \\
u(0, x)=u_{0}(x), \quad x \in \Omega \\
u(t, x)=\phi(t, x), \text { in }(-h, 0) \times \Omega
\end{array}\right.
$$

which has been studied in [5] and [8] (see also [6, 7, 14, 23, 19, 15] for the two-dimensional case).

In this note we prove, in particular, that under suitable assumptions, from the family of solutions $\left\{u^{(N)}\left(\cdot ; u_{0}, \phi\right): N>0\right\}$, we can extract a sequence $\left\{u^{\left(N_{j}\right)}\left(\cdot ; u_{0}, \phi\right): j=1,2, \ldots\right\}$, with $N_{j} \rightarrow+\infty$, which converges in an adequate sense to a weak solution of (1.2). We thus extend the result obtained in [1] for the case without delays.

The structure of the paper is the following. In Section 2 we give some preliminaries on the abstract framework to deal with the problems, and the basic assumptions under which well-possedness of the approximating problems hold, and so that additional estimates can be obtained. In Section 
3 we establish our main result on the convergence of a subsequence of solutions of approximating problems to one weak solution of the problem without modification. Finally, a result about the case when different delays may appear in the approximating problems is also discussed.

\section{Preliminaries}

To set our problem in the abstract framework, we consider the following usual abstract spaces (see [13] and [24, 25]):

$$
\mathcal{V}=\left\{u \in\left(C_{0}^{\infty}(\Omega)\right)^{3}: \operatorname{div} u=0\right\},
$$

$H=$ the closure of $\mathcal{V}$ in $\left(L^{2}(\Omega)\right)^{3}$ with inner product $(\cdot, \cdot)$ and associate norm $|\cdot|$, where for $u, v \in$ $\left(L^{2}(\Omega)\right)^{3}$,

$$
(u, v)=\sum_{j=1}^{3} \int_{\Omega} u_{j}(x) v_{j}(x) \mathrm{d} x,
$$

$V=$ the closure of $\mathcal{V}$ in $\left(H_{0}^{1}(\Omega)\right)^{3}$ with scalar product $((\cdot, \cdot))$ and associate norm $\|\cdot\|$, where for $u, v \in\left(H_{0}^{1}(\Omega)\right)^{3}$,

$$
((u, v))=\sum_{i, j=1}^{3} \int_{\Omega} \frac{\partial u_{j}}{\partial x_{i}} \frac{\partial v_{j}}{\partial x_{i}} \mathrm{~d} x .
$$

We we will use $\|\cdot\|_{*}$ for the norm in $V^{\prime}$ and $\langle\cdot, \cdot\rangle$ for the duality pairing between $V$ and $V^{\prime}$. Finally, we will identify every $u \in H$ with the element $f_{u} \in V^{\prime}$ given by

$$
\left\langle f_{u}, v\right\rangle=(u, v) \text { for all } v \in V .
$$

It follows that $V \subset H \subset V^{\prime}$, where the injections are dense and compact.

Now we define

$$
b(u, v, w)=\sum_{i, j=1}^{3} \int_{\Omega} u_{i} \frac{\partial v_{j}}{\partial x_{i}} w_{j} d x,
$$

for all measurable functions $u, v, w$ defined on $\Omega$ with values in $\mathbb{R}^{3}$ for which the integrals in the right-hand member of the above equality are finite. In particular, $b$ is a trilinear continuous form on $V \times V \times V$.

We denote

$$
b_{N}(u, v, w)=F_{N}(\|v\|) b(u, v, w), \quad \forall u, v, w \in V .
$$

The form $b_{N}$ is linear in $u$ and $w$, but it is nonlinear in $v$. Evidently we have $b_{N}(u, v, v)=0$, for all $u, v \in V$. Moreover, from the properties of $b$ (e.g. see [20] or [24]), and the definition of $F_{N}$, one easily obtains the existence of a constant $C_{1}>0$ only dependent on $\Omega$ such that

$$
\begin{gathered}
\left|b_{N}(u, v, w)\right| \leq C_{1}|u|^{1 / 4}\|u\|^{3 / 4}|v|^{1 / 4}\|v\|^{3 / 4}\|w\|, \quad \forall u, v, w \in V, \\
\left|b_{N}(u, v, w)\right| \leq N C_{1}\|u\|\|w\|, \quad \forall u, v, w \in V .
\end{gathered}
$$

Thus, if we denote

$$
\left\langle B_{N}(u, v), w\right\rangle=b_{N}(u, v, w), \quad \forall u, v, w \in V
$$


we have

$$
\begin{gathered}
\left\|B_{N}(u, v)\right\|_{*} \leq C_{1}|u|^{1 / 4}\|u\|^{3 / 4}|v|^{1 / 4}\|v\|^{3 / 4}, \quad \forall u, v \in V, \\
\left\|B_{N}(u, v)\right\|_{*} \leq N C_{1}\|u\|, \quad \forall u, v \in V .
\end{gathered}
$$

We also consider $A: V \rightarrow V^{\prime}$ defined by $\langle A u, v\rangle=((u, v))$. Denoting $D(A)=\left(H^{2}(\Omega)\right)^{3} \cap V$, then $A u=-P \Delta u, \forall u \in D(A)$, is the Stokes operator $\left(P\right.$ is the ortho-projector from $\left(L^{2}(\Omega)\right)^{3}$ onto $H$ ).

We recall (see [21]) that there exists a constant $C_{2}>0$ depending only on $\Omega$ such that

$$
|b(u, v, w)| \leq C_{2}\|u\|\left\|v \left|\left\|\left.w\right|^{1 / 2}\right\| w \|^{1 / 2}, \quad \forall u, v, w \in V,\right.\right.
$$

and

$$
|b(u, v, w)| \leq C_{2}|u||| v|||A w|, \quad \forall u \in H, v \in V, w \in D(A) .
$$

Moreover, we assume given a mapping $G:(0, T) \times H \rightarrow H$ such that

c1) $G(\cdot, u):(0, T) \rightarrow H$ is measurable, $\forall u \in H$,

c2) there exists a nonnegative function $g \in L^{p}(0, T)$ for some $1 \leq p \leq+\infty$, and a nondecreasing function $L:(0,+\infty) \rightarrow(0,+\infty)$, such that for all $R>0$ if $|u|,|v| \leq R$, then

$$
|G(t, u)-G(t, v)| \leq L(R) g^{1 / 2}(t)|u-v|,
$$

a.e. $t \in(0, T)$, and

c3) there exists a nonnegative function $f \in L^{1}(0, T)$, such that for any $u \in H$,

$$
|G(t, u)|^{2} \leq g(t)|u|^{2}+f(t), \quad \text { a.e. } t \in(0, T) .
$$

Finally, we suppose $\phi \in L^{2 p^{\prime}}(-h, 0 ; H)$ and $u_{0} \in H$, where $1 / p+1 / p^{\prime}=1$.

In this situation, we consider a delay function $\rho \in C^{1}([0, T])$ such that $0 \leq \rho(t) \leq h$ for all $t \in[0, T]$, and there exists a constant $\rho_{*}$ satisfying

$$
\rho^{\prime}(t) \leq \rho_{*}<1 \quad \forall t \in[0, T] .
$$

Definition 2.1 Let $u_{0} \in H$ and $\phi \in L^{2 p^{\prime}}(-h, 0 ; H)$ be given. A weak solution of (1.1) is a function $u \in L^{2 p^{\prime}}(-h, T ; H) \cap L^{2}(0, T ; V) \cap L^{\infty}(0, T ; H)$ such that

$$
\begin{aligned}
& (u(t), w)+v \int_{0}^{t}((u(s), w)) d s+\int_{0}^{t} b_{N}(u(s), u(s), w) d s \\
= & \left(u_{0}, w\right)+\int_{0}^{t}(G(s, u(s-\rho(s))), w) d s,
\end{aligned}
$$

for all $t \in[0, T]$ and all $w \in V$, and coincides with $\phi(t)$ a.e. in $(-h, 0)$.

Remark 2.1 The definition of a weak solution of (1.2) is the analogous of Definition 2.1, but with $b$ instead of $b_{N}$. 
Remark 2.2 If $u$ is a weak solution of (1.1) and we define $\tilde{g}(t)=g\left(\theta^{-1}(t)\right)$, where $\theta:[0, T] \rightarrow$ $[-\rho(0), T-\rho(T)]$ is the differentiable and strictly increasing function given by $\theta(s)=s-\rho(s)$, then, by c3), we obtain

$$
\begin{aligned}
& \int_{0}^{T}|G(t, u(t-\rho(t)))|^{2} d t \\
\leq & \frac{1}{1-\rho_{*}} \int_{-\rho(0)}^{T-\rho(T)} \tilde{g}(t)|u(t)|^{2} d t+\int_{0}^{T} f(t) d t,
\end{aligned}
$$

and therefore, taking into account the facts that $\tilde{g} \in L^{p}(-\rho(0), T-\rho(T))$ and $u \in L^{2 p^{\prime}}(-h, T ; H) \cap$ $L^{\infty}(0, T ; H)$, we have that $G(\cdot, u(\cdot-\rho(\cdot)))$ belongs to $L^{2}(0, T ; H)$. Thus, as $u \in L^{2}(0, T ; V)$ and satisfies the equation

$$
u^{\prime}(t)+v A u(t)+B_{N}(u(t), u(t))=G(t, u(t-\rho(t)))
$$

in $\mathcal{D}^{\prime}\left(0, T ; V^{\prime}\right)$, as a consequence of (2.4), $u^{\prime} \in L^{2}\left(0, T ; V^{\prime}\right)$, and consequently (see [25]) $u \in$ $C([0, T] ; H)$ and satisfies the energy equality

$$
|u(t)|^{2}+2 v \int_{s}^{t}\|u(r)\|^{2} d r=|u(s)|^{2}+2 \int_{s}^{t}(G(r, u(r-\rho(r))), u(r)) d r,
$$

for all $0 \leq s, t \leq T$.

In [4], the following theorem of existence and uniqueness of solutions of (1.1) was proved.

Theorem 2.1 Assume that conditions c1)-c3) are satisfied, and that $u_{0} \in H$ and $\phi \in L^{2 p^{\prime}}(-h, 0 ; H)$ are given. Then, there exists a unique weak solution $u=u\left(\cdot ; u_{0}, \phi\right)$ of $(1.1)$ which is, in fact, a strong solution in the sense that

$$
u \in C([\varepsilon, T] ; V) \cap L^{2}(\varepsilon, T ; D(A)),
$$

for all $0<\varepsilon<T$. Moreover, if $u_{0} \in V$, then

$$
u \in C([0, T] ; V) \cap L^{2}(0, T ; D(A)) .
$$

\section{Convergence to weak solutions of Navier-Stokes Equations}

The main result of this paper is the following.

Theorem 3.1 Assume that conditions c1)-c3) are satisfied, and let $\left\{u^{\left(N_{k}\right)}=u^{\left(N_{k}\right)}\left(\cdot ; u_{0}^{\left(N_{k}\right)}, \phi^{\left(N_{k}\right)}\right): k=\right.$ $1,2, \ldots\}$, where $N_{k} \rightarrow+\infty$ as $k \rightarrow+\infty$, be a sequence of weak solutions of (1.1) with $N=N_{k}$, and with initial data such that $u_{0}^{\left(N_{k}\right)} \rightarrow u_{0}$ weakly in $H, \phi^{\left(N_{k}\right)} \rightarrow \phi$ strongly in $L^{\frac{2 p}{2 p-1}}(-h, 0 ; H)$ as $k \rightarrow+\infty$, and the sequence $\left\{\phi^{\left(N_{k}\right)}: k=1,2, \ldots\right\}$ is bounded in $L^{2 p^{\prime}}(-h, 0 ; H)$.

Then, there exists a subsequence $\left\{u^{\left(N_{j}\right)}: j=1,2, \ldots\right\} \subset\left\{u^{\left(N_{k}\right)}: k=1,2, \ldots\right\}$, with $N_{j} \rightarrow+\infty$, which converges weakly-star in $L^{\infty}(0, T ; H)$, weakly in $L^{2}(0, T ; V)$, weakly-star in $L^{2 p^{\prime}}(-h, T ; H)$, strongly in $L^{\frac{2 p}{2 p-1}}(-h, T ; H)$, and strongly in $L^{2}(0, T ; H)$, to a weak solution $u$ of $(1.2)$. 
Proof. By Remark 2.2, we know that each of these solutions $u^{\left(N_{k}\right)}$ satisfies the energy equality

$$
\frac{1}{2} \frac{d}{d t}\left|u^{\left(N_{k}\right)}(t)\right|^{2}+v\left\|u^{\left(N_{k}\right)}(t)\right\|^{2}=\left(G\left(t, u^{\left(N_{k}\right)}(t-\rho(t))\right), u^{\left(N_{k}\right)}(t)\right), \quad \text { a.e. } t \in(0, T)
$$

and therefore, taking into account c3) and that $\lambda_{1}\left|u^{\left(N_{k}\right)}(t)\right|^{2} \leq\left\|u^{\left(N_{k}\right)}(t)\right\|^{2}$, where $\lambda_{1}$ is the first eigenvalue of the Stokes operator, we obtain

$$
\frac{d}{d t}\left|u^{\left(N_{k}\right)}(t)\right|^{2}+v\left\|u^{\left(N_{k}\right)}(t)\right\|^{2} \leq \frac{1}{v \lambda_{1}}\left(g(t)\left|u^{\left(N_{k}\right)}(t-\rho(t))\right|^{2}+f(t)\right) \quad \text { a.e. } t \in(0, T) .
$$

Consequently, integrating between 0 and $t$, using the function $\tilde{g}$ defined in Remark 2.2, and denoting $\tilde{g}(s)=0$ for all $s>T-\rho(T)$, we obtain

$$
\begin{aligned}
& \left|u^{\left(N_{k}\right)}(t)\right|^{2}+v \int_{0}^{t}\left\|u^{\left(N_{k}\right)}(s)\right\|^{2} d s \\
\leq & \left|u_{0}^{N_{k}}\right|^{2}+\frac{1}{v \lambda_{1}\left(1-\rho_{*}\right)} \int_{0}^{t} \tilde{g}(s)\left|u^{\left(N_{k}\right)}(s)\right|^{2} d s \\
& +\frac{1}{v \lambda_{1}\left(1-\rho_{*}\right)} \int_{-\rho(0)}^{0} \tilde{g}(s)\left|\phi^{\left(N_{k}\right)}(s)\right|^{2} d s+\frac{1}{v \lambda_{1}} \int_{0}^{T} f(s) d s,
\end{aligned}
$$

for all $t \in[0, T]$.

From the assumptions there exists a constant $C>0$ such that

$$
\left|u_{0}^{N_{k}}\right| \leq C \quad \text { and } \quad\left\|\phi^{\left(N_{k}\right)}\right\|_{L^{2 p^{\prime}(-h, 0 ; H)}} \leq C \text {, for all } k .
$$

Therefore, from (3.5) we obtain

$$
\left|u^{\left(N_{k}\right)}(t)\right|^{2}+v \int_{0}^{t}\left\|u^{\left(N_{k}\right)}(s)\right\|^{2} d s \leq K_{T}+\frac{1}{v \lambda_{1}\left(1-\rho_{*}\right)} \int_{0}^{t} \tilde{g}(s)\left|u^{\left(N_{k}\right)}(s)\right|^{2} d s,
$$

for all $t \in[0, T]$, where

$$
K_{T}=C^{2}+\frac{C^{2}}{v \lambda_{1}\left(1-\rho_{*}\right)}\|\tilde{g}\|_{L^{p}(-\rho(0), 0)}+\frac{1}{v \lambda_{1}} \int_{0}^{T} f(s) d s .
$$

Thus, by the Gronwall lemma,

$$
\left|u^{\left(N_{k}\right)}(t)\right|^{2} \leq K_{T} \exp \left(\frac{1}{v \lambda_{1}\left(1-\rho_{*}\right)} \int_{0}^{T} \tilde{g}(s) d s\right)=: C_{T},
$$

for all $t \in[0, T]$.

From (3.6)-(3.8), we obtain that the sequence $\left\{u^{\left(N_{k}\right)}\right\}$ is bounded in the space $L^{2 p^{\prime}}(-h, T ; H) \cap$ $L^{\infty}(0, T ; H) \cap L^{2}(0, T ; V)$. Moreover, taking into account $(2.3)$ and the equation satisfied by $u^{\left(N_{k}\right)}$, we deduce that the sequence of time derivatives $\frac{d}{d t} u^{\left(N_{k}\right)}$ is bounded in $L^{4 / 3}\left(0, T ; V^{*}\right)$. Thus, using in particular the Aubin-Lions compactness theorem (see [13]), there exists a subsequence $u^{\left(N_{j}\right)}$ of the 
$u^{\left(N_{k}\right)}$ which converges to a function $u$ weakly-star in $L^{\infty}(0, T ; H)$, weakly in $L^{2}(0, T ; V)$, weakly-star in $L^{2 p^{\prime}}(-h, T ; H)$, strongly in $L^{\frac{2 p}{2 p-1}}(-h, T ; H)$ and strongly in $L^{2}(0, T ; H)$, and such that $\mid u^{\left(N_{j}\right)}(t)-$ $u(t) \mid \rightarrow 0$ a.e. in $(-h, T), u^{\left(N_{j}\right)} \rightarrow u$ a.e. in $(-h, T) \times \Omega$, and $\frac{d}{d t} u^{\left(N_{k}\right)}$ converges to $\frac{d}{d t} u$ weakly in $L^{4 / 3}\left(0, T ; V^{*}\right)$. Moreover, we can assume, without loss of generality, that there exists a function $z \in L^{\frac{2 p}{2 p-1}}(-h, T)$ such that

$$
\left|u^{\left(N_{j}\right)}(t)\right| \leq z(t) \quad \text { a.e. } t \in(-h, T) .
$$

Now, we are going to prove that this limiting function $u$ is a weak solution of (1.2).

Evidently, as $\phi^{\left(N_{j}\right)} \rightarrow \phi$ strongly in $L^{\frac{2 p}{2 p-1}}(-h, 0 ; H)$, we have that $u(t)=\phi(t)$ a.e. $t \in(-h, 0)$. Thus, by the density of $D(A)$ into $V$, to finish the proof it is enough to see that for all $w \in D(A)$, the function $u$ satisfies

$$
\begin{aligned}
& (u(t), w)+v \int_{0}^{t}((u(s), w)) d s+\int_{0}^{t} b(u(s), u(s), w) d s \\
= & \left(u_{0}, w\right)+\int_{0}^{t}(G(s, u(s-\rho(s))), w) d s \quad t \in[0, T] .
\end{aligned}
$$

Let us fix $w \in D(A)$. We know that for all $j$ one has

$$
\begin{aligned}
& \left(u^{\left(N_{j}\right)}(t), w\right)+v \int_{0}^{t}\left(\left(u^{\left(N_{j}\right)}(s), w\right)\right) d s+\int_{0}^{t} b_{N_{j}}\left(u^{\left(N_{j}\right)}(s), u^{\left(N_{j}\right)}(s), w\right) d s \\
= & \left(u_{0}^{\left(N_{j}\right)}, w\right)+\int_{0}^{t}\left(G\left(s, u^{\left(N_{j}\right)}(s-\rho(s))\right), w\right) d s \quad t \in[0, T] .
\end{aligned}
$$

Evidently, by the assumptions,

$$
\left(u_{0}^{\left(N_{j}\right)}, w\right) \rightarrow\left(u_{0}, w\right) .
$$

Also, as $u^{\left(N_{j}\right)}$ converges to $u$ weakly in $L^{2}(0, T ; V)$, we have that

$$
\left(\left(u^{\left(N_{j}\right)}, w\right)\right) \rightarrow((u, w)) \quad \text { in } L^{2}(0, T) .
$$

Now, observe that by c2), a.e. $t \in(0, T)$ the function $G(t, \cdot): H \rightarrow H$ is continuous; thus, as $\left|u^{\left(N_{j}\right)}(s)-u(s)\right| \rightarrow 0$ a.e. in $(-h, T)$, we have that

$$
G\left(t, u^{\left(N_{j}\right)}(t-\rho(t))\right) \rightarrow G(t, u(t-\rho(t))) \quad \text { in } H \text { a.e. in }(0, T) .
$$

On the other hand, by c3) and (3),

$$
\left|G\left(t, u^{\left(N_{j}\right)}(t-\rho(t))\right)\right| \leq g^{1 / 2}(t) z(t-\rho(t))+f^{1 / 2}(t) \quad \text { a.e. in }(0, T) .
$$

Thus, by dominated convergence,

$$
G\left(\cdot, u^{\left(N_{j}\right)}(\cdot-\rho(\cdot))\right) \rightarrow G(\cdot, u(\cdot-\rho(\cdot))) \quad \text { in } L^{1}(0, T ; H) .
$$

We claim that

$$
b_{N_{j}}\left(u^{\left(N_{j}\right)}(\cdot), u^{\left(N_{j}\right)}(\cdot), w\right) \rightarrow b(u(\cdot), u(\cdot), w) \quad \text { weakly in } L^{1}(0, T) .
$$


This can be seen as follows. First, we observe that by the boundedness of the sequence $\left\{u^{\left(N_{j}\right)}\right\}$ in $L^{2}(0, T ; V)$, we have (see $[1$, Lem.12]) that

$$
F_{N_{j}}\left(\left\|u^{\left(N_{j}\right)}(\cdot)\right\|\right) \rightarrow 1 \quad \text { in } L^{2}(0, T)
$$

On the other hand, reasoning as in [13, pp.76-77], one obtains that

$$
b\left(u^{\left(N_{j}\right)}(\cdot), u^{\left(N_{j}\right)}(\cdot), w\right) \rightarrow b(u(\cdot), u(\cdot), w) \quad \text { weakly in } L^{2}(0, T) .
$$

Moreover, from the boundedness of the sequence $\left\{u^{\left(N_{j}\right)}\right\}$ in $L^{2}(0, T ; V) \cap L^{\infty}(0, T ; H)$ and (2), we see that the sequence $\left\{b\left(u^{\left(N_{j}\right)}(\cdot), u^{\left(N_{j}\right)}(\cdot), w\right)\right\}$ is bounded in $L^{2}(0, T)$. Then, from (3) and (3), we can deduce (3.14).

Finally, as $u^{\left(N_{j}\right)} \rightarrow u$ in $L^{2}(0, T ; H)$, we have that

$$
\left(u^{\left(N_{j}\right)}, w\right) \rightarrow(u, w) \quad \text { in } L^{2}(0, T) .
$$

From (3.10)-(3.14) and (3.15), we deduce that (3.9) holds a.e. $t \in(0, T)$. Now observe that $u \in$ $L^{\infty}(0, T ; H)$, and also, due to the fact that $u^{\prime} \in L^{4 / 3}\left(0, T ; V^{\prime}\right)$, we have that $u \in C\left([0, T] ; V^{\prime}\right)$. Thus, $u$ is weakly continuous as a function from $[0, T]$ into $H$ (see [22]), and therefore (3.9) holds for all $t \in[0, T]$.

With a slight modification of the proof of Theorem 3.1, it is possible to prove the following result.

Theorem 3.2 Assume that $G(\cdot, \cdot):(-h, T+h) \times H \rightarrow H$ is continuous, and conditions $c 2)$ and c3) hold with $p=1$. Let $\left\{\rho^{\left(N_{k}\right)}: k=1,2, \ldots\right\} \subset C^{1}([-h, T+h])$, where $N_{k} \rightarrow+\infty$ as $k \rightarrow+\infty$, be a sequence of functions such that $0 \leq \rho^{\left(N_{k}\right)}(t) \leq h$ for all $t \in[-h, T+h]$ and any $k \geq 1$, there exist constants $\rho_{*}<1$ and $\rho^{*} \geq 0$ such that

$$
-\rho^{*} \leq\left(\rho^{\left(N_{k}\right)}\right)^{\prime}(t) \leq \rho_{*} \quad \text { for all } t \in[-h, T+h] \text { and any } k \geq 1,
$$

and

$$
\rho^{\left(N_{k}\right)} \rightarrow \bar{\rho}(t) \quad \text { uniformly in } C^{1}([-h, T+h]) \text { as } k \rightarrow+\infty .
$$

For each $k \geq 1$, let us denote $u^{\left(N_{k}\right)}$, the corresponding weak solution of (1.1) with $N=N_{k}$, $\rho=\rho^{\left(N_{k}\right)}$, and with initial data $\left(u_{0}^{\left(N_{k}\right)}, \phi^{\left(N_{k}\right)}\right)$ such that $u_{0}^{\left(N_{k}\right)} \rightarrow u_{0}$ weakly in $H, \phi^{\left(N_{k}\right)} \rightarrow \phi$ strongly in $L^{2}(-h, 0 ; H)$ as $k \rightarrow+\infty$, and the sequence $\left\{\phi^{\left(N_{k}\right)}: k=1,2, \ldots\right\}$ is bounded in $L^{\infty}(-h, 0 ; H)$.

Then, there exists a subsequence $\left\{u^{\left(N_{j}\right)}: j=1,2, \ldots\right\} \subset\left\{u^{\left(N_{k}\right)}: k=1,2, \ldots\right\}$, with $N_{j} \rightarrow+\infty$, which converges weakly-star in $L^{\infty}(-h, T ; H)$, weakly in $L^{2}(0, T ; V)$, and strongly in $L^{2}(-h, T ; H)$, to a weak solution $u$ of (1.2) with $\rho=\bar{\rho}$.

Proof. The beginning of the proof follows the same steps as in Theorem 3.1. Indeed it only requires to be adapted slightly to deal with the different delays, but the uniform estimates can be obtained similarly.

However, although it is possible to obtain a uniform estimate for $\left|G\left(t, u^{\left(N_{j}\right)}\left(t-\rho^{\left(N_{j}\right)}(t)\right)\right)\right|$, since now $z \in L^{\infty}(-h, T)$, the pass to the limit from $G\left(t, u^{\left(N_{j}\right)}\left(t-\rho^{\left(N_{j}\right)}(t)\right)\right)$ to $G(t, u(t-\bar{\rho}(t)))$ is not so clear, since the time arguments of $u^{\left(N_{j}\right)}$ and $u$ are different, and the convergence only seems to hold in $V^{\prime}$ but not in $H$. 
This can be circumvented in the following way. To conclude that $u$ is a solution for (1.2) it only remains to prove that

$$
\int_{0}^{t}\left(G\left(s, u^{\left(N_{j}\right)}\left(s-\rho^{\left(N_{j}\right)}(s)\right)\right), w\right) d s \rightarrow \int_{0}^{t}(G(s, u(s-\bar{\rho}(s))), w) d s \quad \text { as } j \rightarrow+\infty,
$$

since the rest of the convergences in the approximating equations toward the expected limit follow analogously as in Theorem 3.1.

Using the change of variable $\theta_{j}(s):=s-\rho^{\left(N_{j}\right)}(s)=r,(3.16)$ is equivalent to prove that

$$
\begin{aligned}
& \int_{-\rho^{\left(N_{j}\right)}(0)}^{t-\rho^{\left(N_{j}\right)}(t)} \frac{1}{1-\left(\rho^{\left(N_{j}\right)}\right)^{\prime}\left(\theta_{j}^{-1}(r)\right)}\left(G\left(\theta_{j}^{-1}(r), u^{\left(N_{j}\right)}(r)\right), w\right) d r \\
\rightarrow & \int_{-\bar{\rho}(0)}^{t-\bar{\rho}(t)} \frac{1}{1-\bar{\rho}^{\prime}\left(\bar{\theta}^{-1}(r)\right)}\left(G\left(\bar{\theta}^{-1}(r), u(r)\right), w\right) d r \quad \text { as } j \rightarrow+\infty,
\end{aligned}
$$

where $\bar{\theta}(s):=s-\bar{\rho}(s)$.

Indeed, the proof of the above convergence again will follows from the dominated convergence theorem (observe that now the functions $u^{\left(N_{j}\right)}$ and $u$ are written in the same time values), thanks to the continuity of $G$, and if we finally check that $\theta_{j}^{-1}(r) \rightarrow \bar{\theta}^{-1}(r)$ as $j \rightarrow+\infty$, for almost all $r \in(-h, T)$. Actually, we claim that

$$
\theta_{j}^{-1}(r) \rightarrow \bar{\theta}^{-1}(r) \quad \text { as } j \rightarrow+\infty \text {, for all } r \in(-h, T) .
$$

Indeed, if it is not so, there exist values $\hat{r} \in(-h, T)$ and $\varepsilon>0$, and a subsequence relabelled $\left\{\theta_{j}^{-1}\right\}$, such that $\left|\theta_{j}^{-1}(\hat{r})-\bar{\theta}^{-1}(\hat{r})\right| \geq \varepsilon$ for all $j$. Then, there are two possibilities.

Case 1: For a subsequence, again relabelled $\left\{\theta_{j}^{-1}\right\}$,

$$
\theta_{j}^{-1}(\hat{r}) \geq \bar{\theta}^{-1}(\hat{r})+\varepsilon \quad \text { for all } j .
$$

Due to the strict monotonicity of $\bar{\theta}$ and $\bar{\theta}^{-1}$ and the fact that $\hat{r}<T$, then $\bar{\theta}^{-1}(\hat{r})<T+h$. Taking if necessary a smaller value $\varepsilon>0$, we may assume that $\bar{\theta}^{-1}(\hat{r})+\varepsilon \in[-h, T+h]$. We now make the composition of the expressions in (3.19) with $\theta_{j}$. This leads to $\hat{r}=\theta_{j}\left(\theta_{j}^{-1}(\hat{r})\right) \geq \theta_{j}\left(\bar{\theta}^{-1}(\hat{r})+\varepsilon\right)$. Passing to the limit when $j \rightarrow+\infty$ and using that $\bar{\theta}$ is strictly increasing, we obtain that

$$
\hat{r} \geq \bar{\theta}\left(\bar{\theta}^{-1}(\hat{r})+\varepsilon\right)>\bar{\theta}\left(\bar{\theta}^{-1}(\hat{r})\right)=\hat{r}
$$

which is a contradiction.

Case 2: For a subsequence, again relabelled $\left\{\theta_{j}^{-1}\right\}$,

$$
\bar{\theta}^{-1}(\hat{r}) \geq \theta_{j}^{-1}(\hat{r})+\varepsilon \quad \text { for all } j .
$$

We claim that

$$
\theta_{j}^{-1}(\hat{r})+\varepsilon \leq T+h \text { for all } j
$$

if $\varepsilon>0$ is small enough. 
Indeed, by the mean value theorem, for a fixed $\delta \in(0, T-\hat{r})$, we have that $\theta_{j}^{-1}(\hat{r}+\delta)=\theta_{j}^{-1}(\hat{r})+$ $\delta\left(\theta_{j}^{-1}\right)^{\prime}\left(r_{\hat{r}, \delta}\right)$, with $r_{\hat{r}, \delta} \in(\hat{r}, \hat{r}+\delta)$. On other hand, from the assumptions on $\rho^{\left(N_{j}\right)}$, we have that $\min _{[\hat{r}, \hat{r}+\delta]}\left(\theta_{j}^{-1}\right)^{\prime}(\cdot) \geq 1 /\left(1+\rho^{*}\right)$. Taking if necessary a smaller value $\varepsilon>0$ so that $\delta /\left(1+\rho^{*}\right) \geq \varepsilon$, and using the monotonicity of the functions $\theta_{j}^{-1}$, we conclude that

$$
\begin{aligned}
\theta_{j}^{-1}(\hat{r})+\varepsilon & \leq \theta_{j}^{-1}(\hat{r})+\frac{\delta}{1+\rho^{*}} \\
& \leq \theta_{j}^{-1}(\hat{r}+\delta) \\
& <\theta_{j}^{-1}(T) \\
& \leq T+h .
\end{aligned}
$$

This proves (3.21) and we can compose in (3.20) with $\theta_{j}$, whence the monotonicity of all $\theta_{j}$ implies that

$$
\theta_{j}\left(\bar{\theta}^{-1}(\hat{r})\right) \geq \theta_{j}\left(\theta_{j}^{-1}(\hat{r})+\varepsilon\right) .
$$

Using again the mean value theorem in the right hand side, $\theta_{j}\left(\theta_{j}^{-1}(\hat{r})+\varepsilon\right) \geq \hat{r}+\left(1-\rho_{*}\right) \varepsilon$, whence passing to the limit when $j \rightarrow+\infty$ above, we conclude that

$$
\hat{r} \geq \hat{r}+\left(1-\rho_{*}\right) \varepsilon,
$$

which is a contradiction.

This gives (3.18), which jointly with the assumptions on $\rho^{\left(N_{j}\right)}$ and $\bar{\rho}$, and $G$, and the pointwise convergence for almost all times of $u^{\left(N_{j}\right)}$ to $u$, allow us to pass to the limit by the dominated convergence theorem, obtaining (3.17), whence the result follows.

\section{References}

[1] T. Caraballo, P. E. Kloeden, and J. Real, Unique strong solutions and V-attractors of a three dimensional system of Globally Modified Navier-Stokes equations, Adv. Nonlinear Stud. 6 (2006), 411-436.

[2] T. Caraballo, P. E. Kloeden, and J. Real, Addendum to the paper "Unique strong solutions and V-attractors of a three dimensional system of Globally Modified Navier-Stokes equations", Advanced Nonlinear Studies 6 (2006), 411-436, Adv. Nonlinear Stud. 10 (2010), 245-247.

[3] T. Caraballo, P. E. Kloeden, and J. Real, Invariant measures and statistical solutions of the globally modified Navier-Stokes equations, Discrete Contin. Dyn. Syst. Ser. B 10 (2008), 761-781.

[4] T. Caraballo, A. M. Márquez-Durán, and J. Real, Three dimensional system of globally modified NavierStokes equations with delay, Internat. J. Bifur. Chaos Appl. Sci. Engrg. 20 (2010), 2869-2883.

[5] T. Caraballo and J. Real, Navier-Stokes equations with delays, R. Soc. Lond. Proc. Ser. A Math. Phys. Eng. Sci. 457 (2001), 2441-2453.

[6] T. Caraballo and J. Real, Asymptotic behaviour of two-dimensional Navier-Stokes equations with delays, R. Soc. Lond. Proc. Ser. A Math. Phys. Eng. Sci. 459 (2003), 3181-3194.

[7] T. Caraballo and J. Real, Attractors for 2D-Navier-Stokes models with delays, J. Differential Equations 205 (2004), 271-297.

[8] M. J. Garrido-Atienza and P. Marín-Rubio, Navier-Stokes equations with delays on unbounded domains, Nonlinear Anal. 64 (2006), 1100-1118. 
[9] P. E. Kloeden, T. Caraballo, J. A. Langa, J. Real, and J. Valero, The three dimensional globally modified Navier-Stokes equations, in Mathematical Problems in Engineering Aerospace and Sciences, Vol. 3, Chapter 2, Eds.: S. Sivasundaram, J. Vasundhara Devi, Zahia Drici and Farzana Mcrae, Cambridge Scientific Publishers, 2009.

[10] P. E. Kloeden, J. A. Langa, and J. Real, Pullback V-attractors of a three dimensional system of nonautonomous globally modified Navier-Stokes equations: Existence and finite fractal dimension, Commun. Pure Appl. Anal. 6 (2007), 937-955.

[11] P. E. Kloeden, P. Marín-Rubio, and J. Real, Equivalence of Invariant measures and Stationary Statistical solutions for the autonomous globally modified Navier-Stokes equations, Commun. Pure Appl. Anal. 8 (2009), 785-802.

[12] P. E. Kloeden and J. Valero, The weak connectedness of the attainability set of weak solutions of the $3 D$ Navier-Stokes equations, Proc. Roy. Soc. London A 463 (2007), 1491-1508.

[13] J. L. Lions, Quelques méthodes de résolution des problèmes aux limites non linéaires, Dunod, GauthierVillars, Paris, 1969.

[14] P. Marín-Rubio and J. Real, Attractors for 2D-Navier-Stokes equations with delays on some unbounded domains, Nonlinear Anal. 67 (2007), 2784-2799.

[15] P. Marín-Rubio and J. Real, Pullback attractors for 2D-Navier-Stokes equations with delays in continuous and sub-linear operators, Discrete Contin. Dyn. Syst. 26 (2010), 989-1006.

[16] P. Marín-Rubio, A. M. Márquez, and J. Real, Three dimensional system of globally modified Navier-Stokes equations with infinite delays, Discrete Contin. Dyn. Syst. Ser. B 14 (2010), 655-673.

[17] P. Marín-Rubio, A. M. Márquez, and J. Real, Pullback attractors for globally modified Navier-Stokes equations with infinite delays, Discrete Contin. Dyn. Syst. 31 (2011), 779-796.

[18] P. Marín-Rubio, J. Real, and J. Valero, Pullback attractors for a 2D-Navier-Stokes model in an infinite delay case, Nonlinear Anal. 74 (2011), 2012-2030.

[19] G. Planas and E. Hernández, Asymptotic behaviour of two-dimensional time-delayed Navier-Stokes equations, Discrete Contin. Dyn. Syst. 21 (2008), 1248-1288.

[20] J. C. Robinson, Infinite-dimensional dynamical systems, Cambridge University Press, Cambridge, 2001.

[21] M. Romito, The uniqueness of weak solutions of the globally modified Navier-Stokes equations, Adv. Nonlinear Stud. 9 (2009), 425-427.

[22] W. A. Strauss, On Continuity of Functions with Values in Various Banach Spaces, Pacific J. Math. 19 (1966), 543-551.

[23] T. Taniguchi, The exponential behavior of Navier-Stokes equations with time delay external force, Discrete Contin. Dyn. Syst. 12 (2005), 997-1018.

[24] R. Temam, Navier-Stokes equations, Theory and Numerical Analysis, AMS Chelsea Publishing, Providence, Rhode Island, 2001.

[25] R. Temam, Navier-Stokes Equations and Nonlinear Functional Analysis, Second Edition, SIAM, Philadelphia, 1995. 